\title{
Kimura's Disease with Atypical Musculoskeletal Presentation
}

\author{
A Hafiz, MSur Ortho, A Yusuf*, MSur Ortho, I Rosmaliza****, MS ORL, \\ N Premchandran**, MSur Ortho, R Kalavathy***, MPath \\ Department of Orthopaedics, Traumatology \& Rehabilitation, International Islamic University Malaysia, \\ Kuantan, Pahang \\ *Department of Orthopaedic, Hospital Sultan Haji Ahmad Shah, Temerloh, Malaysia \\ **Department of Orthopaedic, Hospital Tengku Ampuan Afzan Kuantan, Malaysia \\ ***Department of Pathology, Hospital Tengku Ampuan Afzan Kuantan, Malaysia \\ **** Department of ORL, Hospital Tengku Ampuan Afzan Kuantan, Malaysia
}

\begin{abstract}
We would like to highlight an unusual clinical presentation of Kimura's disease, a rare, benign, idiopathic condition, usually seen as swelling and lesions of the head and neck region and commonly affecting young men of Asian descent. It is rare for this disorder to present with lesions on other parts of the body, especially the lower limbs. We report a 27 year-old man who presented with a large mass located at the inguinal region and extending down to the upper thigh. Due to the large size of the mass $(28 \times 18 \mathrm{~cm})$, our provisional diagnosis was a soft tissue sarcoma. Open biopsy showed that the lesion was benign.
\end{abstract}

Key Words:

Kimura's Disease, Atypical presentation

\section{INTRODUCTION}

Kimura's disease was first described in 1937 by Kimm and Szeto in China. It is a rare, idiopathic condition that usually affects young men of Asian origin. The disease usually presents with swelling and lesions in the head and neck region. The lesions may involve the subcutaneous soft tissue, major salivary glands and lymph nodes. It is rare for this disease to affect other parts of the body. Diagnosis is confirmed by biopsy and histopathology.

\section{CASE REPORT}

A twenty-seven-year-old man presented in January 2000 with a painless, gradually enlarging mass in the left groin of three years duration. The swelling dd not interfere with his routine daily activity. There was no history of trauma, local infection or insect bite. Physical examination revealed a mass at the anterior aspect of the left groin extended down to left upper thigh, measuring approximately $28 \mathrm{~cm} \mathrm{x} 18 \mathrm{~cm}$.
The mass was not attached to the overlying skin and the surface of the lesion showed hyperpigmentation. It was a nontender, firm, oval, soft tissue swelling with indistinct boundaries, mobile with a lobulated surface. The superficial inguinal lymph node was palpable $(2 \times 2 \mathrm{~cm})$. There was no other swelling noted elsewhere. Sensation and pulses were intact distally. (Figure 1)

Plain radiographs of the left groin showed soft tissue swelling overlying the left hip. The underlying bone and adjacent hip joint were normal and there was no periosteal reaction. Magnetic resonance imaging (MRI) of the left groin showed soft tissue swelling with adipose tissue infiltration (Figure 2). Imaging studies are not diagnostic in these cases, but help to delineate the extent of disease. The following laboratory results were obtained: haemoglobin, $15.7 \mathrm{~g} / \mathrm{dl}$; white blood cell count, $23.1 \times 10^{3} / \mu \ell$; and, platelets, $333 \times 10^{3} / \mu \ell$. The renal profile and liver function results were normal. The erythrocytes sedimentation (ESR) rate was $11.0 \mathrm{~mm} /$ first hr. and urine analysis was normal.

An open biopsy revealed a benign lesion. Microscopic analysis of the lesion sample showed follicular hyperplasia with dilated sinuses and marked proliferation of high endothelial vessels. There was prominent eosinophilia; in fact, a number of the follicles showed eosinophilia abscess with occasional necrosis. There were also nodules of marked vascular proliferation with surrounding lymphoid cells and eosinophilia.. There was however, no evidence of malignant change (Figure 3). A diagnosis of Kimura's disease was made.

The patient was treated with high dose steroids for six weeks. The swelling become smaller, regressing to $21 \mathrm{~cm} \times 14 \mathrm{~cm}$ after medication. The mass was then resected and sent for biopsy. On gross examination, the lesion measured $18.5 \mathrm{x}$ $11.5 \times 5 \mathrm{~cm}$ and the weight was 560 grams. It was welldefined and firm in consistency. Sectioning revealed large

Corresponding Author: Dr Ahmad Hafiz Zulkifly, Department of Orthopaedics, Traumatology \& Rehabilitation, Kulliyyah of Medicine, International Islamic University Malaysia, Jalan Hospital, 25100 Kuantan, Pahang Email: hafiz_ortho@yahoo.com 

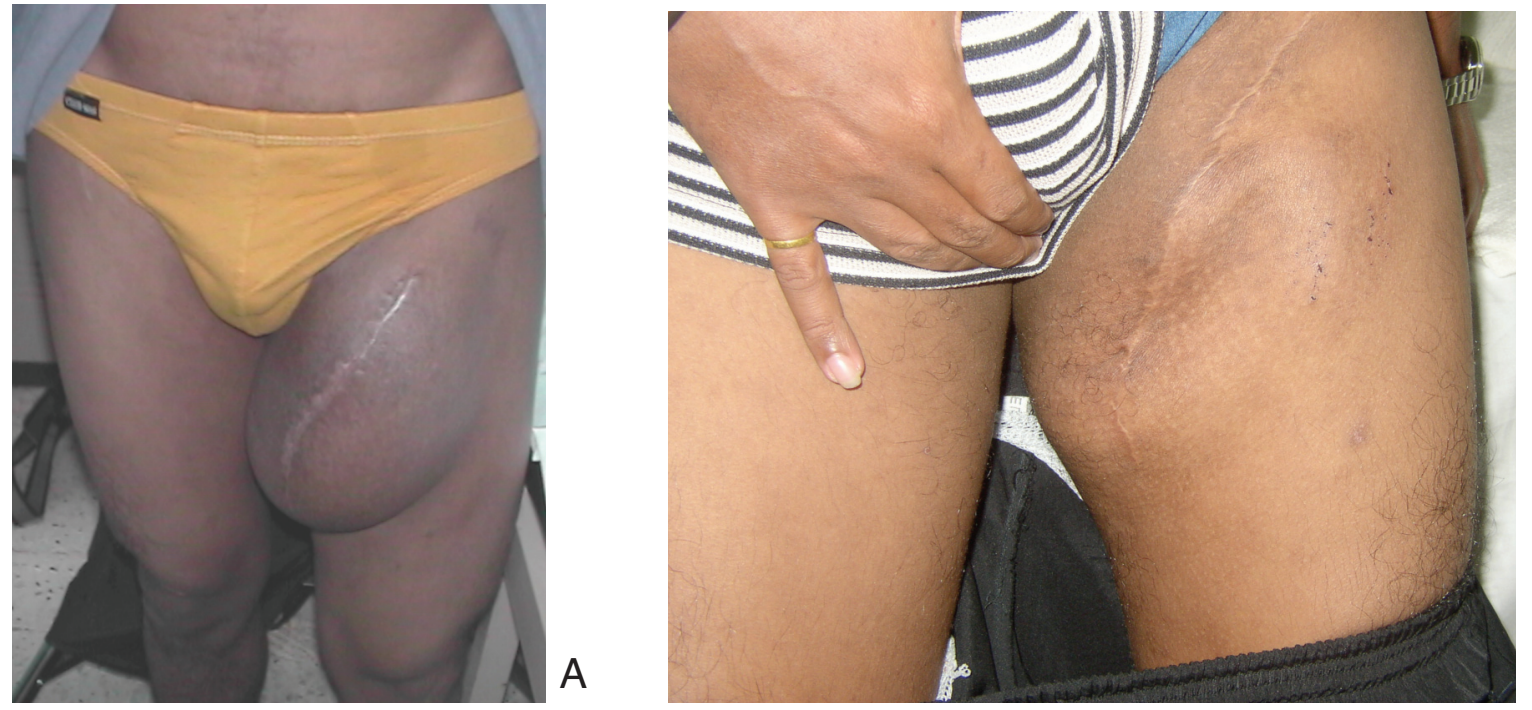

B

Fig. 1: Picture taken after open biopsy showed large swelling at the left groin (A) (May 2000). After Resection (B) (July 2005)

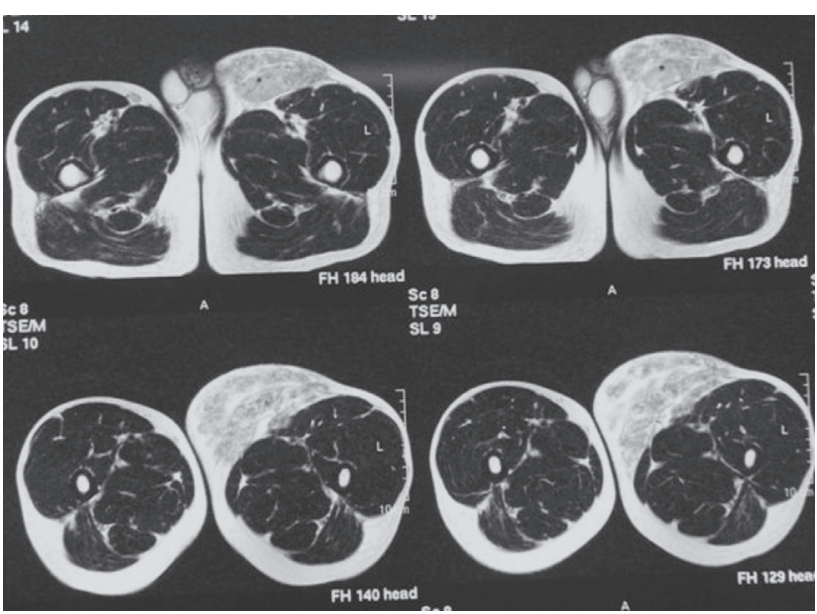

Fig. 2: Magnetic resonance imaging (MRI) of his left groin showed soft tissue swelling with fat tissue infiltration.

amounts of oedematous adipose tissue intertwined with blood vessels. There were no obvious haemorrhage or necrosis. Histological findings were consistent with initial biopsy and confirmed the diagnosis of a Kimura's disease.

The patient recovered well post operatively. He was prescribed a second six week course of high dose of steroid medication. Currently, at seven years post surgical resection he has no evidence of recurrence except occasional intense itchiness of the affected area. He takes anti-histamine medication symptomatic relief of itching.

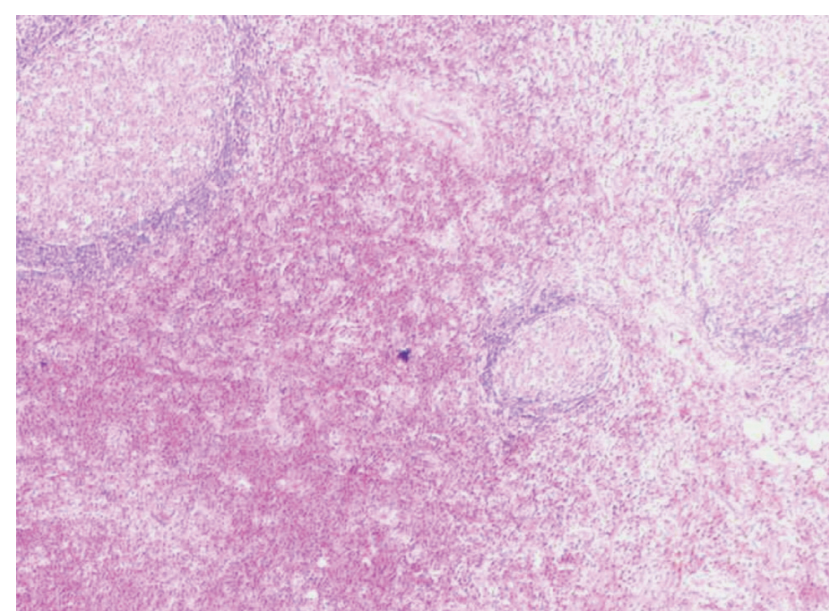

Fig. 3: Follicular hyperplasia with dilated sinuses and marked proliferation of high endothelial vessels $(X 10)$. A number of the follicles show eosinophilia abscess with occasional necrosis.

\section{DISCUSSION}

Kimura's Disease is a rare lesion that mimics soft tissue tumours. First described in 1937 by Kimm and Szeto in China as "eosinophilic hyperplastic granuloma", in 1948 Kimura reported the disease as "unusual granulation and hyperplastic changes of lymphatic tissue"; from that point the disease became known as Kimura's disease. This disease is endemic in part of Asia and mainly affects young Asian males.

The aetiology of Kimura's disease is unknown although the presence of eosinophilia and elevated serum IgE levels suggests an allergic or hypersensitivity process. The intense 
itchiness of the affected area seen in this case, is not mentioned in previous published reports, so seems very unusual for the disease. It may relate to the hypersensitivity process in the pathology of the disease. The clinical course of Kimura's disease is benign. In about $12 \%$ of patient, there may be associated renal disease, usually presenting as proteinuria. The subcutaneous masses of Kimura's disease are usually found in the head and neck region, sometimes affecting the parotid or minor salivary glands. Involvement of the groin region and limbs, as seen in the current report, is very rare.

Plain radiograph is not useful in diagnostic purposes in this disease. Som and Biller demonstrated the usefulness of computer tomography and magnetic resonance images in the investigation of in their report. Although imaging studies are not diagnostic, they may help delineate the extent of disease. The diagnosis of Kimura's disease requires biopsy or excision of the lesion. Histologically, the tissues show a reactive prominent germinal centre composed of three elements as seen in this patient: cellular, fibrocollagenous, and vascular.
Therapeutic options for Kimura's disease have been reported as follows. Resection of the tumour mass may be effective in permanently eradicating the mass if the entire lesion can be removed, but recurrence is common. Local irradiation has also been shown to be effective in shrinking lesions, but is generally not advocated in younger patients. Systemic and intralesional corticosteroid administration have been shown to reduce the size of the lesion, but the tumour tends to recur when these drugs are discontinued. In the present case, the patient was treated with a combination of resection of the lesion and oral corticosteroids. To date, there is no recurrence noted at seven years post treatment. In selected patients, it may be advisable to take a more conservative approach, treating only if the lesion continues to grow or becomes symptomatic.

In the case reported herein, Kimura's disease showed good response with surgical excision and oral steroid therapy. This was a rare presentation of a benign lesion probably due to hypersensitivity process. Intense itchiness was noted in this case and treated with anti-histamine medication. 


\section{REFERENCES}

1. Chusid MJ, Rock AL, Sty JR, Oechler HW, Beste DJ. Kimura's disease: an unusual cause of cervical tumour. Arch Dis Child 1997; 77: 153-4.

2. Ingrams DR, Stafford ND, Creagh TM. Angiolymphoid hyperplasia with eosinophilia. J Laryngol Otol. 1995; 109: 262-4.

3. Karavattathayyil SJ, Krause JR. Kimura's disease. A case report. Ear, Nose throat J; 2000; 79: 195-9.

4. Som PM, Biller HF. Kimura disease involving parotid gland and cervical nodes: CT and MR findings. J Comput Assist Tomogr 1992; 16: 320-2. 